\title{
Effects of Combined Exercise Training on Balance of Hemiplegic Stroke Patients
}

\author{
Won Seob Shin, PT, $\mathrm{PhD}^{1)}$, Seung Won Lee, PT, PhD ${ }^{1)}$, Yong Woo Lee, PT, DC, MSc ${ }^{1)}$, \\ Sung Bum CHOI, DC, MSC ${ }^{2)}$, CHANG Ho SONG, PT, PhD ${ }^{1)}$ \\ 1) Department of Physical Therapy, Sahmyook University: 26-21, Gongneung2-dong, Nowon-gu, Seoul, \\ 139-742, Republic of Korea. \\ TEL: +82 2-3399-1630,FAX: +82 2-3399-1638,E-mail: chsong@syu.ac.kr \\ ${ }^{2)}$ Choisooyong Oriental Medicine Clinic
}

J. Phys. Ther. Sci.

23: 639-643, 2011

\begin{abstract}
Purpose] The purpose of this study was to investigate the effects of combined exercise training with aerobic and functional strengthening exercises on balance ability of hemiplegic stroke patients. [Subjects] The subjects were 21 patients with chronic stroke who were attending a rehabilitation center. [Methods] Participants were randomly allocated to two groups: a combined exercise training group $(n=11)$ and a conventional exercise group $(n=10)$. Both groups did 60 minutes exercise per day, 5 times a week for four weeks. [Results] Both groups showed significant improvements in static and dynamic balances, and the combined exercise showed greater improvement in dynamic balance. [Conclusion] The result of this study suggests that combined exercise training with functional strengthening exercise and aerobic exercise was effective at improve static and dynamic balance ability, and was more effective than conventional exercise at improving dynamic balance. This suggests that combined exercise training can be prescribed for stroke patients to reduce their risk of falls and lead to independent ADL.
\end{abstract}

Key words: Stroke, Combined exercise training, Static balance, Dynamic balance

(This article was submitted Mar. 10, 2011, and was accepted Apr. 5, 2011)

\section{INTRODUCTION}

A stroke occurs as a result of brain damage caused by cerebral infarction or hemorrhage. The majority, 73 88\%, of cerebral infarction patients have impaired sensory motor ability on the side opposite to the brain damage appearing as hemiplegia of the arms and legs or either ${ }^{1}$. Most survivors of stroke have decreased capacity to perform activities of daily living (ADL) because of a combination of sensory, motor, cognitive and emotional impairments ${ }^{2}$. Of all the possible sensorimotor deficits of stroke, damaged postural control has the greatest impact on ADL independence and gait $^{3)}$.

Hemiplegic patients have decreased balance control ability. Especially, the postural sway in static positions is more than twice that of healthy subjects of the same age group, which consequences for safety ${ }^{4)}$. Balance is described as the ability to maintain equilibrium in a gravitational field by keeping or returning the center of body mass over its base of support ${ }^{5)}$. Dynamic balance is a voluntary response which maintains the position in response to an external perturbation ${ }^{6}$. Stroke patients with both motor and sensory deficits show high incidences of falls both during rehabilitation and thereafter due to loss of postural control ${ }^{7}$.

Many methods for improving the balance of stroke patients have been studied. An exercise program which promote weight bearing by the hemiplegic lower extremity was prescribed for stroke survivors by physical therapists ${ }^{8,9)}$. Functional strengthening training enhances the interaction of the nervous and muscular systems, maximizing functional regulation to improve $\mathrm{ADL}^{10)}$. Task oriented functional training on weight-bearing was especially effective ${ }^{11,12)}$. In Frenceshini and colleagues' study ${ }^{13)}$, stroke patients' trunk control ability improved after treadmill gait training, which to improved balance ability. When stroke patients performed standing in functional strengthening training, the rate of weight bearing on the affected side increased, improving their balance ability ${ }^{11)}$. In the study of Katz-Leuer and his colleagues ${ }^{14)}$, the balance of stroke patients was improved by bicycle training.

Based on the available evidence, the best therapeutic approach to influence the speed or extent of standing balance recovery in the subacute phase of stroke is controversial $^{15)}$. Physiological approaches and intervention methods have been studied for rehabilitation of stroke patients ${ }^{16)}$. Some previous studies have shown clinical effects of combined exercise. Combined aerobic and resistance exercises increased muscle strength, and decreased glycated hemoglobin and fasting glucose significantly in type 2 diabetes ${ }^{17}$. In a study of male with coronary artery disease, exercise endurance increased and 
Table 1. Characteristic of subjects

\begin{tabular}{lcccccc}
\hline Group & $\mathrm{N}$ & Sex (male/female) & Hemiplegic side (Rt./Lt.) & Age (years) & Height (cm) & Weight $(\mathrm{kg})$ \\
\hline Combined Ex. & 11 & $5 / 6$ & $8 / 3$ & $58.1(4.6)$ & $160.6(6.6)$ & $65.2(8.3)$ \\
Conventional Ex. & 10 & $3 / 7$ & $5 / 5$ & $57.3(4.4)$ & $164.5(7.1)$ & $65.0(7.5)$ \\
\hline
\end{tabular}

NOTE. Values are frequency or mean (SD).

fat mass decreased significantly in a combined aerobic and strength training group compared with a group which received only aerobic training ${ }^{18)}$. Combined exercise training that mixes strengthening, aerobic, balance, and gait training has been studied. This type of training increased muscle strengthening and balance ability to prevent falls ${ }^{19)}$. However, combined exercise training research is incomplete and needs more research for stroke patients ${ }^{20)}$. Although there are many comparative studies which used single exercise groups, conventional treatment groups, and nonexercise control groups for stroke patients, few comparative studies of the effects of combined exercise have been performed, especially with stroke patients as subjects. It is not clear whether or not the combination of exercise for muscle strength improvement and aerobic exercise, which are effective in gait training, is more effective than conventional rehabilitation exercise. Therefore the purpose of this study was to investigate the effects of combined exercise training with aerobic and functional strengthening exercises on balance of hemiplegic stroke patients.

\section{SUBJECTS AND METHODS}

The subjects of this study were 21 patients with stroke who were attending a rehabilitation center (Table 1). The inclusion criteria were the following: subjects who were between 6 months and 5 years since diagnosis of stroke, subjects with hemiplegia of the lower extremities. Patients who met the following criteria were excluded: subjects who could not ride a bicycle or perform functional exercise due to arthritis, low-back pain, or degenerative joint disease; subjects who were receiving medical treatment due to other symptoms; and subjects who could not follow the instructions due to low perceptive abilities, cognitive disorder, or communication disorder ${ }^{14,21)}$. All subjects provided their written informed consent prior to participation in the study.

The twenty-one subjects were randomly allocated to one of into two groups: a combined exercise training group and a conventional exercise group. The combined group which consisted of 11 subjects did exercise combined with aerobic and functional strengthening exercises for balance. The conventional exercise group which consisted of 10 subjects did conventional exercise. Each group exercised for 60 minutes per day, 5 times a week, for four weeks.

In the combined exercise training group the first exercise was $30 \mathrm{~min}$ of functional strength training, consisting of six sub-categories: bridge exercise, lifting toes and ankles, sitting and standing, stretching out the arms while standing, step exercise, and stairs exercise. Bridge exercise is lifting pelvis using the legs, from bending hips and knees with supine. Lifting toes and ankles exercise is dorsiflexion and plantar flexion of the hemiplegic leg in the sitting position. Sitting and standing is standing from sitting and sitting again until the hip touches chair. Stretching out the arms while standing is stretching out the arms upward, downward, right-side, left-side, and diagonally. Step exercise is shifting of weight bearing to a leg on a step. The hemiplegic leg and non-hemiplegic leg are placed in turn on the step, and the location of step alternates from the front to one side of the subject. Stairs exercise is walking up stairs with the hemiplegic leg supporting the body weight and walking down stairs with the non-hemiplegic leg support body weight. Before exercise, 5 minutes warming-up exercise of breathing exercise and stretching were conducted. Each exercise was repeated at medium intensity without fatigue ten to fifteen times ${ }^{11)}$. The second exercise for the combined exercise training group was aerobic exercise. Treadmill walking and riding a bicycle were conducted for fifteen minutes each. Treadmill walking started at $0.5 \mathrm{~m} / \mathrm{s}$ and the initial 5 minutes was on adaption period. In the next 10 minutes walking velocity increased or walking was done with less support from eht hand-rail ${ }^{22)}$. A stationary bicycle was used for the bicycle riding exercise. At the beginning a patient started with a velocity which he/ she could feel comfortable with. As time went by the velocity was increased. The intensity of the two aerobic exercises was determined by checking the heat rate. Polar (Polar Vantage NV, Polar Electro Oy, Finland) was used for monitoring the heart rate. Heart rate was not allowed to exceed $40 \%$ of the heart rate reserve. Heart rate reserve was calculated using age and maximum heart rate. Resting heart rate was evaluated at the same time in the morning and reevaluated on the first day of each week. The intensity of exercise was adjusted following a change in resting heart rate $^{14)}$. Aerobic exercise was conducted with the assistance of a caregiver or family member under the supervision of a physical therapist. The conventional training was conducted for 60 minutes a day, the same duration as the combined exercise group. Special instructions were not given to the physical therapist in charge, and the conventional training was done as usual. The therapist focused on re-educating normal movement during functional activities that were meaningful to the patients. Training was composed of balance exercise, posture control exercise, and gait exercise. Keeping normal movement of the pelvis for balance and posture control was emphasized. The therapist judged that working on increasing anterior and posterior pelvic tilt would improve weight transfer and hip extension during gait, leading to improvements in selective distal control of the knee and the foot. Trunk control and alignment can affect muscle tone, range of motion, and control of the limb. 
Table 2. Comparison of static balance

\begin{tabular}{|c|c|c|c|c|}
\hline Measures & & & Combined Ex. $(n=11)$ & Conventional Ex. $(n=10)$ \\
\hline \multirow{9}{*}{$\begin{array}{l}\text { Eye Open } \\
(\mathrm{cm})\end{array}$} & \multirow{3}{*}{ ML } & Pretest & $30.2(11.0)$ & $30.6(11.5)$ \\
\hline & & Posttest & $27.6(10.0) *$ & $28.3(10.1) *$ \\
\hline & & Post-Pre & $2.6(3.4)$ & $2.3(3.0)$ \\
\hline & & Pretest & $30.4(5.5)$ & $29.3(5.0)$ \\
\hline & \multirow{2}{*}{ AP } & Posttest & $28.2(5.5) *$ & $26.8(4.4) *$ \\
\hline & & Post-Pre & $2.2(1.6)$ & $2.5(1.5)$ \\
\hline & & Pretest & $51.1(14.5)$ & $53.2(13.4)$ \\
\hline & \multirow[t]{2}{*}{$\mathrm{TS}$} & Posttest & $46.8(12.3) *$ & $48.7(11.7) *$ \\
\hline & & Post-Pre & $4.3(4.0)$ & $4.5(3.7)$ \\
\hline \multirow{9}{*}{$\begin{array}{l}\text { Eye Closed } \\
(\mathrm{cm})\end{array}$} & \multirow{3}{*}{ ML } & Pretest & $45.6(25.9)$ & $50.8(27.3)$ \\
\hline & & Posttest & $38.6(19.4) *$ & $41.6(19.9) *$ \\
\hline & & Post-Pre & $7.0(9.1)$ & $9.2(11.5)$ \\
\hline & & Pretest & $33.9(6.5)$ & $34.0(7.0)$ \\
\hline & \multirow[t]{3}{*}{ AP } & Posttest & $30.9(5.2) *$ & $30.3(6.9) *$ \\
\hline & & Post-Pre & $3.0(3.4)$ & $3.7(4.6)$ \\
\hline & & Pretest & $70.3(32.7)$ & $64.2(27.1)$ \\
\hline & \multirow[t]{2}{*}{ TS } & Posttest & $62.9(25.3) *$ & $56.6(20.5) *$ \\
\hline & & Post-Pre & $11.0(7.5)$ & $7.7(10.0)$ \\
\hline
\end{tabular}

NOTE. Values are mean(SD). Abbreviation: ML; medial to lateral Sway, AP; anterior to posterior Sway, TS; total sway, Pre-Post; pretest-posttest. ${ }^{*} \mathrm{p}<0.05$ from pretest.

For the upper limbs, treatment was conducted focusing on movement of the scapular. For balance exercise, weight transfer exercise and reaching exercise were alternately performed on the affected side and the unaffected side in the sitting or standing position. Bridging exercise was performed to strengthen the trunk muscles. Selective movement of each joint of the shoulder, elbow, knee, and ankle joint was performed to facilitate upper and lower limb movement. For gait exercise, training in weight transfer during gait was conducted by planting the unaffected side foot at the front and back of the body. Gait training was divided between the stance phase and the swing phase, and exercises for each phase were performed. In addition, stair climbing practice and gait training for crossing obstacles were conducted. Patients did not do the same exercise every training day but suitable exercises were selected according to the goals of each patient and the therapist. The intensity of each exercise was decided by the therapist considering each patient's capacity for exercise ${ }^{13)}$. Static and dynamic were evaluated. Static balance was measured on a force platform. The force platform (PDM Multifunction Force Measuring Plate, Zebris, Germany, 2004) operates with 1504 capacitive force sensors arranged in a $32 \times 47 \mathrm{~cm}$ matrix. It allows analysis of the static and dynamic force and pressure distribution under the feet when standing. Each sensor works independently. The force platform measures parameters such as center of pressure (COP), limits of stability, shear forces, center of gravity, center of mass, and the derivatives of these parameters ${ }^{15}$ ). The range of measured pressure is $1-120 \mathrm{~N} / \mathrm{cm}^{2}$, the sampling rate is $2-5$ $\mathrm{Hz}$ in static mode and $90 \mathrm{~Hz}$ in dynamic mode, and the accuracy is $\pm 5 \%$. Subjects were instructed to stand on the platform comfortably, and position their put feet at the same location for re-evaluation. Arms were held comfortably each side of the body. Evaluations were made with the eyes open and closed. With eye open a patient gazed at a $15 \mathrm{~cm}$ diameter circle, $3 \mathrm{~m}$ ahead. Data were collected three times, and three minutes resting time was given to minimize the effect of muscle fatigue.

Dynamic balance was measured using the Berg balance scale. The Berg balance scale (BBS) is used to evaluate aged people and subjects with neurologic deficits. It measures abilities of maintaining posture, controlling posture by voluntary movement, and responses to environmental changes. BBS is a clinical balance test of 14 common tasks: standing from sitting, standing without support, sitting without leaning backward, sitting from standing, movement between chairs, standing with eye closed, standing with both legs together without support, stretching out the arms while standing, lifting something from the floor, turning to the right and left sides, turning around on the spot, putting the feet on a step in turn, standing and putting one foot in front of the other foot, standing on one leg. Performance of each task is graded on a five-point ordinal scale ranging from 0 to 4 , with higher scores reflecting better balance. The maximum score of all the test items is $56^{23}$.

Statistical analysis was performed using SPSS Statistical Package, version 15.0 (SPSS Inc, Chicago, IL). Data was analyzed by utilizing separate $2 \times 2$ [Group (Combined Ex., Conventional Ex.)] $\times$ Test (pre, post)] two-way ANOVA with repeated measures for all other variables. The level of statistical significance was chosen as 0.05 .

\section{RESULTS}

At the start of the intervention, there were no significant differences between the two groups in medial to lateral (ML) sway distance, anterior to posterior (AP) sway distance, and total sway (TS) distance among the static balance parameters. After completion of the 4-week 
Table 3. Comparison of dynamic balance

\begin{tabular}{llcc}
\hline Measures & & Combined Ex. $(\mathrm{n}=11)$ & Conventional Ex. $(\mathrm{n}=10)$ \\
\hline BBS (point) & Pretest & $42.3(8.7)$ & $41.5(8.4)$ \\
& Posttest & $45.6(7.5) *$ & $43.4(8.5) *$ \\
& Post-Pre & $3.4(1.6)$ & $1.9(1.5) * \S$ \\
\hline
\end{tabular}

NOTE. Values are mean $(\mathrm{SD})$. Group $=$ main effect for Group factor; Test $=$ main effect for Test factor; Group $\times$ Test $=$ interaction. ${ }^{*} \mathrm{p}<0.05$ from pretest. ${ }^{\S} \mathrm{p}<0.05$ from Combined Ex.

intervention, both groups showed significant improvements in static balance under both the eyes open and eyes closed conditions. There were no significant Group $\times$ Test interactions for AP, ML and TS distance for the eyes open and eyes closed conditions. However, significant main effects for Test were observed for all of the static balance parameters $(p<0.05)$. Results for the eyes open condition show respective decreases in AP, ML, and TS distances of $2.6 \pm 3.4,2.2 \pm 1.6$, and $4.3 \pm 4.0 \mathrm{~cm}$ for the combined exercise group and $2.3 \pm 3.0,2.5 \pm 1.5$, and $9.2 \pm 11.5 \mathrm{~cm}$ for conventional exercise group. The results for the eyes closed condition showed respective decreases in AP, ML, and TS distance of $7.0 \pm 9.1,3.0 \pm 3.4$, and $11.0 \pm 7.5 \mathrm{~cm}$ for the combined exercise group and $9.2 \pm 11.5,3.7 \pm 4.6$, and 7.7 $\pm 10.0 \mathrm{~cm}$ for the conventional exercise group. These results indicate that both groups achieved significant improvements in static balance over the 4 week period (Table 2).

The pre-intervention dynamic balance of the two groups was not significantly different. The BBS of each group increased significantly $(\mathrm{p}<0.05)$ post-intervention. There was a significant Group $\times$ Test interaction for BBS $(p<0.05)$ and a significant main effect for Test was observed for BBS $(p<0.05)$. However, no significant main effect of Group was observed for BBS. The results showed an increase in BBS of $3.4 \pm 1.6$ points for the combined exercise group and $1.9 \pm$ 1.5 points for the conventional exercise group. These data indicate that both groups achieved significant improvements in dynamic balance over the 4-week intervention period (Table 3).

\section{DISCUSSION}

This study was conducted to analyze the effects of combined by group comparing static and dynamic balance. In functional strengthening exercise with aerobic training the sway distance with eyes open significantly decreased by $2.63 \mathrm{~cm}$ in ML sway, by $2.24 \mathrm{~cm}$ in AP sway, and by 4.32 $\mathrm{cm}$ in TS. These results show that the combined exercise program was effective for static balance. The decrease in static balance with eye closed was statistically significant $(p<0.05)$. Fast shifting of the body center changes static balance modulation to dynamic balance modulation. The ability to maintain balance can be improved by task-specific training ${ }^{24)}$. Balance consists of static and dynamic balance. Static balance is the ability to stand on fixed ground without swaying, and dynamic balance is the ability to maintain balance in response to external perturbation ${ }^{25)}$. Postural sway occurs even in standing, and the degree of sway depends on the distance separating the feet, or the location of the feet ${ }^{26)}$. Some studies have analyzed COP displacement as a measure of postural sway. It is the point location of the resultant vertical ground reaction force. It reflects the trajectory of the center of body mass and the amount of torque applied at the support surface to control body mass accelerations ${ }^{5)}$. In a study of 46 stroke patients within 80 days of stroke onset, Walker et al. ${ }^{27)}$ reported postural sway velocity was $4.9 \mathrm{~mm} / \mathrm{s}$ with eyes open, and $10.7 \mathrm{~mm} / \mathrm{s}$ with eyes closed, and these velocities were 3 times higher than those of healthy persons.

In this study BBS was used to evaluate balance ability. The results show that in the combined exercise group, BBS was increased by 3.36 and in the conventional exercise group by 1.90 . The changes in the Berg balance scale in each group were statistically significant $(\mathrm{p}<0.05)$. The difference between the groups was also statistically significant $(\mathrm{p}<0.05)$. Weiss et al. ${ }^{28)}$ reported that high intensity strength training improved strength and functional performance by $12 \%$ after stroke. Wing et al. ${ }^{29)}$ also reported that BBS was increased from 46.7 to 47.2 after intensive repetitive training, locomotor training, and functional strength training. The study of Ducan et al. ${ }^{30)}$ showed that BBS was increased by 4.36 , from 42.8 to 47.16 , after an exercise program targeting flexibility, strength, balance, endurance, and upper-extremity function. It is important to note that the mean post-intervention BBS score was 45.64 , which is over the cutoff score, 45 out of 56 , for an increased risk of falling ${ }^{29}$. Thus, it is possible that patients who are at a lower risk of falling may not need to improve balance in order to improve functional mobility.

With the addition of a handrail or increased body weight support, the percentage of single limb support time on the paretic limb increased and temporal symmetry improved ${ }^{31)}$. Also cycling training with body weight support, as an automatically symmetric exercise, activated the paretic limb muscles, and increased the ability of motion of each side. These interventions improved the balance ability of stroke patients ${ }^{14)}$.

The ability to control body weight on support both the paretic limb and non-paretic limb is equally important in ADL and it is a goal of rehabilitation training ${ }^{3)}$. The result of this study suggests that combined exercise training can be clinically prescribed for stroke patients to reduce the risk of falls and lead to independent ADL.

\section{REFERENCES}

1) Tangeman PT, Banaitis DA, Williams AK: Rehabilitation of chronic stroke patients: changes in functional performance. Arch Phys Med Rehabil, 1990, 71: 876-880. 
2) Hochstenbach J, Donders R, Mulder T, et al.: Long-term outcome after stroke: a disability-orientated approach. Int J Rehabil Res, 1996, 19: 189-200.

3) Fong KN, Chan CC, Au DK: Relationship of motor and cognitive abilities to functional performance in stroke rehabilitation. Brain Inj, 2001, 15: 443-453.

4) Nichols DS: Balance retraining after stroke using force platform biofeedback. Phys Ther, 1997, 77: 553-558.

5) Niam S, Cheung W, Sullivan PE, et al.: Balance and physical impairments after stroke. Arch Phys Med Rehabil, 1999, 80: 1227-1233.

6) Berg KO, Maki BE, Williams JI, et al.: Clinical and laboratory measures of postural balance in an elderly population. Arch Phys Med Rehabil, 1992, 73: 1073-1080.

7) Pollock A, Baer G, Pomeroy V, et al.: Physiotherapy treatment approaches for the recovery of postural control and lower limb function following stroke. Cochrane Database Syst Rev, 2003: CD001920.

8) Nilsson LM, Nordholm LA: Physical therapy in stroke rehabilitation: bases for Swedish physiotherapists' choice of treatment. Physiother Theory Pract, 1992, 8: 49-55.

9) Carr JH, Mungovan SF, Shepherd RB, et al.: Physiotherapy in stroke rehabilitation: bases for Australian physiotherapists'choice of threatment. Physiother Theory Pract, 1994, 10: 201-209.

10) Kwakkel G, Wagenaar RC, Koelman TW, et al.: Effects of intensity of rehabilitation after stroke. A research synthesis. Stroke, 1997, 28: 1550-1556.

11) Bale M, Strand LI: Does functional strength training of the leg in subacute stroke improve physical performance? A pilot randomized controlled trial. Clin Rehabil, 2008, 22: 911-921.

12) Krebs DE, Scarborough DM, McGibbon CA: Functional vs. strength training in disabled elderly outpatients. Am J Phys Med Rehabil, 2007, 86: 93-103.

13) Franceschini M, Carda S, Agosti M, et al.: Walking after stroke: what does treadmill training with body weight support add to overground gait training in patients early after stroke?: a single-blind, randomized, controlled trial. Stroke, 2009, 40: 3079-3085.

14) Katz-Leurer M, Sender I, Keren O, et al.: The influence of early cycling training on balance in stroke patients at the subacute stage. Results of a preliminary trial. Clin Rehabil, 2006, 20: 398-405.

15) Geurts AC, Nienhuis B, Mulder TW: Intrasubject variability of selected forceplatform parameters in the quantification of postural control. Arch Phys Med Rehabil, 1993, 74: 1144-1150.

16) Morris SL, Dodd KJ, Morris ME: Outcomes of progressive resistance strength training following stroke: a systematic review. Clin Rehabil, 2004, 18:27-39.

17) Maiorana A, O’Driscoll G, Goodman C, et al.: Combined aerobic and resistance exercise improves glycemic control and fitness in type 2 diabetes. Diabetes Research and Clinical Practice, 2002, 56: 115-123

18) Santa-Clara H, Fernhall B, Baptista F, et al.: Effect of a one-year combined exercise training program on body composition in men with coronary artery disease. Metabolism, 2003, 52: 1413-1417.

19) Englund U, Littbrand H, Sondell A, et al.: A 1-year combined weight-bearing training program is beneficial for bone mineral density and neuromuscular function in older women. Osteoporos Int, 2005, 16: 1117-1123.

20) Baker MK, Atlantis E, Fiatarone Singh MA: Multi-modal exercise programs for older adults. Age Ageing, 2007, 36: 375-381.

21) Janssen TW, Beltman JM, Elich P, et al.: Effects of electric stimulationassisted cycling training in people with chronic stroke. Arch Phys Med Rehabil, 2008, 89: 463-469.

22) Sullivan KJ, Brown DA, Klassen T, et al.: Effects of task-specific locomotor and strength training in adults who were ambulatory after stroke: results of the STEPS randomized clinical trial. Phys Ther, 2007, 87: 1580-1602.

23) Berg KO, Wood-Dauphinee SL, Williams JI, et al.: Measuring balance in the elderly: validation of an instrument. Can J Public Health, 1992, 83 Suppl 2: S7-S11.

24) Flynn S, Palma P, Bender A: Feasibility of using the Sony PlayStation 2 gaming platform for an individual poststroke: a case report. J Neurol Phys Ther, 2007, 31: 180-189.

25) Ragnarsdóttir M: The concept of balance. Physiotherapy, 1996, 82: 368-375.

26) Gatev P, Thomas $S$, Kepple T, et al.: Feedforward ankle strategy of balance during quiet stance in adults. J Physiol, 1999, 514: 915-928.

27) Walker C, Brouwer BJ, Culham EG: Use of visual feedback in retraining balance following acute stroke. Phys Ther, 2000, 80: 886-895.

28) Weiss A, Suzuki T, Bean J, et al.: High intensity strength training improves strength and functional performance after stroke. Am J Phys Med Rehabil, 2000, 79: 369-376; quiz 391-364.

29) Wing K, Lynskey JV, Bosch PR: Whole-body intensive rehabilitation is feasible and effective in chronic stroke survivors: a retrospective data analysis. Top Stroke Rehabil, 2008, 15: 247-255.

30) Duncan P, Studenski S, Richards L, et al.: Randomized clinical trial of therapeutic exercise in subacute stroke. Stroke, 2003, 34: 2173-2180.

31) Chen G, Patten C, Kothari DH, et al.: Gait deviations associated with poststroke hemiparesis: improvement during treadmill walking using weight support, speed, support stiffness, and handrail hold. Gait Posture, 2005, 22: $57-62$. 